\section{Migräne und offenes Foramen ovale}

* Reisman AM, Robbins BT, Chou DE, Shnayderman Yugrakh M, Gross G], Privitera L, Nazif T, Sommer RJ. Ticagrelor for Refractory Migraine/ Patent Foramen Ovale (TRACTOR). Neurology 2018; 91: 1010-1017.

Eine offene Studie mit dem Thrombozytenaggregationshemmer Ticagrelor konnte keine eindrückliche Wirksamkeit zeigen. Die Diskussion bleibt aber weiterhin offen.

\section{Zusammenfassung}

Der Zusammenhang zwischen Migräne (insbesondere mit Aura) und offenem Foramen ovale (PFO) ist bereits in vielen Studien untersucht worden. In dieser Studie wurde insbesondere gefragt, ob sich eine Thrombozytenaggregationshemmung mit Ticagrelor, das bei koronarer Herzkrankheit eingesetzt wird, günstig auf Migräne auswirkt, und zwar insbesondere bei Patienten mit Migräne und PFO. Die Studie stützt sich auf Beobachtungen, dass die Thienopyridine Clopidogrel und Prasugrel Migränekopfschmerzen bei Patienten mit PFO reduzierten konnten.

Es wurden insgesamt 40 Patienten mit einem nachgewiesenen PFO und Migräne eingeschlossen. Sie erhielten über 28 Tage offen Ticagrelor $2 \times 90 \mathrm{mg}$. Eine Reduzierung um über $50 \%$ der Kopfschmerztage wurde als Erfolg angesehen. Eine solche Reduzierung zeigten 17 Patienten nach 28 Tagen. Diese wurden 2 weitere Monate ebenfalls mit Ticagrelor $2 \times 90 \mathrm{mg}$ offen behandelt. Responder waren insgesamt jünger und hatten weniger Kopfschmerztage pro Monat während der Baseline als Non-Responder. Es zeigten sich keine Unterschiede bezüglich episodische versus chronische Migräne, bezüglich Aura oder nicht und bezüglich der Größe des PFO. Insgesamt 13 Patienten hatten Nebenwirkungen nach der Einnahme von Ticagrelor, überwiegend Atemstörungen und Präsynkopen. Die Autoren schließen aus ihren Daten, dass Ticagrelor nicht besser wirksam ist als Thienopyridine, aber mehr Nebenwirkungen aufweist und deswegen nicht für kontrollierte Studien in Betracht kommt.

\section{Kommentar}

Der Zusammenhang zwischen Migräne und PFO wird (berechtigterweise) immer noch diskutiert, sowohl hinsichtlich der Pathophysiologie als auch therapeutischer Konsequenzen. Dabei haben zahlreiche große Studien mit einer Ausnahme gezeigt, dass ein PFO bei Migräne mit Aura signifikant häufiger auftritt. Die großen klinischen Studien waren dann allerdings in ihrem primären Endpunkt sämtlich negativ. Daraus wird oft in der Literatur fälschlicherweise geschlossen, dass ein Verschluss des PFO bei Migräne überhaupt nicht wirksam ist. Randomisierte Studien haben jedoch konsistent gezeigt, dass Migräne mit Aura und auch die Aura alleine signifikant reduziert werden durch den Verschluss des PFO. Es ist aber noch unklar, wodurch dieser Zusammenhang begründet ist. In der hier vorgelegten Studie wurden nun Patienten mit Migräne und PFO mit einem Thrombozytenaggregationshemmer untersucht. Würde der Zusammenhang zwischen Migräne und PFO in der Thrombozytenaggregation (mit möglichen zerebralen Mikroembolien) liegen, müsste eine Hemmung ähnlich wie bei anderen Substanzen (Clopidogrel, ASS) zu einer deutlichen Reduzierung der Migräne führen. Die hier vorgelegte Studie konnte aber eine eindrückliche Wirksamkeit von Ticagrelor nicht zeigen. Hierbei ist zu berücksichtigen, dass die Studie extrem klein war und dass die Untergruppe der Responder mit Migräne mit Aura z. B. nur bei $n=7$ lag. Damit lassen sich keine statistischen Analysen durchführen und Rückschlüsse ziehen. Tendenziell $(p=0,13)$ war es auch bei dieser kleinen Fallzahl so, dass Migräne mit Aura besser auf Ticagrelor angesprochen hat als Migräne ohne Aura. Auch anderen Analysen (z. B. in Bezug auf die Größe des PFO) können aus statistischer Sicht nicht sinnvollerweise ausgewertet werden. Insgesamt hilft die Studie in der Diskussion leider nicht weiter. Immerhin werden derzeit neue große Studien geplant, die gezielt erneut den therapeutischen Einfluss eines Verschlusses des PFO auf Migräne mit Aura untersuchen wollen.

Stefan Evers, Coppenbrügge

\section{Insuffiziente Versorgung von Menschen mit Migräne in den USA}

\footnotetext{
**** Lipton RB, Munjal S, Alam A, Buse DC, Fanning KM, Reed ML, Schwedt T], Dodick DW. Migraine in America Symptoms and Treatment (MAST) Study: Baseline Study Methods,

Treatment Patterns, and Gender Differences. Headache 2018; 58: 1408-1426.
}

Die populationsbasierte MAST-Studie aus den USA zeigt, dass viele Patienten mit Migräne keine korrekte Diagnose und keine migränespezifische Behandlung erhalten.

\section{Hintergrund}

Migräne ist sehr verbreitet, führt häufig bei den Betroffenen zu einer deutlichen Beeinträchtigung und Reduktion der Lebensqualität und trotzdem werden Patienten mit Migräne häufig nicht adäquat versorgt, sowohl in Europa als auch in den USA. In der heutigen Zeit der Einführung von CGRP-AK ist die Frage der Qualität der medizinischen Versorgung von Patienten mit Migräne aktuell wie nie zuvor.

\section{Zusammenfassung}

In einer großen internetbasierten Befragung in den USA wurden 95821 Menschen zu Migräne befragt. Identifiziert wurden 15131 Menschen mit Migräne, die mindestens 1 Kopfschmerzattacke in den letzten 3 Monaten erlitten hatten. Eine weitere detaillierte Befragung der 15131 Menschen mit Migräne befasste sich mit Fragen zur Soziodemografie, klinischen Merkmalen von Migräne und medizinischen Versorgung wie zum Beispiel Einnahme von akuter und prophylaktischer Medikation, Arztbesuche etc.

Erwartungsgemäß gab es mehr Frauen als Männer mit Migräne, im Durchschnitt waren die Betroffenen 43 Jahre alt, und überwiegend weiß. Die meisten (ca. $80 \%$ ) waren mindestens einmal im Leben wegen ihrer Kopfschmerzen beim Arzt gewesen. Frauen erhielten die Migränediagnose in $48 \%$ und Männer in $38 \%$. Die Mehrheit (60\%) nahmen OTC-Medikamente, und nur ca. 10-15\% nahmen Triptane zur Akutbehandlung von Kopfschmerzen ein. Pro- 
phylaktische Medikation nahmen lediglich $10-14 \%$ der Betroffenen ein.

Die Autoren schlussfolgern, dass obwohl die meisten Migränepatienten mindestens einmal einen Arzt aufgesucht hatten, die medizinische Versorgung trotzdem insuffizient war.

\section{Kommentar}

Die Studie beweist noch einmal, dass sogar in reichen Ländern wie den USA die Versorgung von Migräne insuffizient ist. Ähnliche Ergebnisse sind mehrfach für die USA aber auch für Europa veröffentlicht worden. Die Problematik ist sehr vielfältig und beginnt mit den Patienten selbst, die in Bezug auf ihre Kopfschmerzen häufig inkonsequent handeln. Aber auch Apotheker, Hausärzte, Neurologen, Schmerztherapeuten und Frauenärzte könnten und sollten mehr tun, um die aktuell sicherlich nicht zufriedenstellende Lage zu verbessern.

Zaza Katsarava, Unna

\section{Migräne-Auslöser und Habituation visuell evozierter Potentiale}

\footnotetext{
**** Lisicki M, Ruiz-Romagnoli E, Piedrabuena R, Giobellina R, Schoenen J, Magis D. Migraine triggers and habituation of visual evoked potentials. Cephalalgia 2018; 38: 988-992.
}

Migränepatienten, bei denen wahrgenommener Stress kein Anfallsauslöser ist, sind möglicherweise eine Untergruppe mit speziellen klinisch-physiologischen Merkmalen im Migränespektrum.

\section{Zusammenfassung}

Mit der Studie soll die Frage nach einer personalisierten Migränebehandlung beantwortet werden. Dabei wird die These zugrunde gelegt, dass spezifische Eigenschaften in Untergruppen von Migränepatienten gefunden werden können, mit denen eine zielgerichtete Behandlung möglich wäre. Die Autoren gehen von folgenden Annahmen aus: a) es gibt eine Reihe von Migräneauslösern; b) Anfallsauslöser sind kein
Teil der IHS-Kriterien, sie charakterisieren aber Subgruppen von Patienten; c) bei Migränepatienten liegt eine fehlende Habituation auf wiederholte gleichförmige Stimulation vor, was jedoch nicht immer und nicht bei jedem Patienten beobachtet werden kann. Vielleicht kann ein unterschiedliches VEP-Habituationsprofil die unterschiedliche Wirkung von Migräneauslösern erklären?

Es handelt sich um eine Querschnittstudie unter Zugrundelegung von 25 Migränepatienten ohne Aura und ohne medikamentöse Prophylaxe, bei denen sowohl eine VEP-Untersuchung als auch ein halbstrukturiertes Interview zu Art und Umfang von Migräneauslösern durchgeführt wurde. Dabei wurde eine Liste vorgegeben, bei denen Stress, Änderung der Ess- und Schlafgewohnheiten, hormonelle Veränderungen, Wetteränderungen, Alkoholkonsum, Geruchs- und Lichtexposition, Rauch, physische Belastung und sexuelle Aktivität aufgeführt waren. Die Patienten mussten angeben, ob die vorgegebenen Auslöser bei ihnen wirksam seien. Bei der VEP-Messung wurde der mittlere Habituationsgradient von 6 Blöcken mit jeweils 100 VEP-Durchgängen bei $\mathrm{Oz}$ auf die Angabe zu den individuellen Triggern bezogen. Bei 4,5 Anfallsauslösern pro Patient wurden Stress, Nahrungskarenz und zu wenig Schlaf am häufigsten als Trigger genannt. Es zeigte sich, dass nur die Patienten mit Stress als Auslöser ein signifikantes Habituationsdefizit hatten, während dies bei den Patienten ohne Stress als Auslöser nicht nachgewiesen wurde. Die anderen Triggerfaktoren hatten keinen signifikanten Einfluss.

Die Arbeitsgruppe diskutiert zunächst die Ergebnisse eines fehlenden Habituationsdefizits in einer Untergruppe, für das keine tragfähige Erklärung gegeben werden kann. Jedoch kann Stress selbst die Amplitude bei der VEP-Messung vergrößern, was durch eine fehlende Habituation erklärt werden kann. Eine gute Erklärung bietet die von der Arbeitsgruppe schon früher vertretene Annahme einer verminderten Prä-Aktivierung in den sensorischen Rindengebieten mit dem Effekt einer größeren kortikalen Aktivierbarkeit. Damit könnte ein intrinsischer Mangel an hemmenden Mechanismen angenommen werden. Dieser Mangel drückt sich in einer geringeren serotoninergen Transmission aus, welche sowohl bei der Migräne als auch bei akuten Stressreaktionen beobachtet werden kann. Der Bezug zu Stress legt Verbindungen zur Mitochondrienfunktion und zu Varianten der CNR1-Gencodierung nahe. Stress muss also nicht unbedingt ein Anfallsauslöser sein. Wenn dies der Fall ist, liegt eine Untergruppe vor, die sich phänotypisch und möglicherweise auch genetisch von der Gruppe der anderen Migränepatienten unterscheidet.

\section{Kommentar}

Die Studie reiht sich ein in ein Profil, welches seit Jahrzehnten Migränepatienten charakterisiert: Hohe Amplituden und fehlende Habituation bei evozierten Potenzialmessungen. Dies ist jedoch nicht zwangsweise immer zu beobachten, weswegen Reliabilitätsuntersuchungen zur Habituation eher fehlschlagen [2], obwohl die zugrunde liegenden Amplitudenmessungen als sehr zuverlässig gewertet werden können (hohe Reliabilität als Beispiel bei langsamen Gleichspannungspotentialen in einer gesunden Stichprobe) [1]. Deswegen muss zwangsläufig eine Moderatorvariable vorliegen, welche die Arbeitsgruppe der vorliegenden Studie in der individuellen Stressreagibilität der Patienten über einen Fragebogen identifiziert hat. Ein phänotypischer Marker kann demnach nur in definierten Untergruppen von Migränepatienten vorliegen, wobei das trennende Kriterium die Art des Reizes ist, der die individuelle Auslösung von Migräneanfällen bewirken kann. Es dürfte deswegen nur noch eine Frage der Zeit sein, dass die IHS-Klassifikation von Kopfschmerzen spezifische Auslösebedingungen von Migräneanfällen als klassifikatorische Kriterien mit aufnimmt.

Peter Kropp, Rostock

Literatur

[1] Kropp P, Kiewitt A, Göbel H, Vetter P, Gerber WD. Reliability and stability of contingent negative variation. Appl Psychophysiol Biofeedback 2000; 25(1): 33-41.

[2] Rauschel V, Ruscheweyh R, Krafczyk S, Straube A. Test-retest reliability of visual-evoked potential habituation. Cephalalgia 2016; 36(9): 831-839. 


\section{Der CGRP-Antikörper}

\section{Galcanezumab zur} Prophylaxe der Migräne: nicht alle Responder sprechen im ersten Monat an

\footnotetext{
*** Nichols R, Doty E, Sacco S, Ruff D, Pearlman E, Aurora SK. Analysis of Initial Nonresponders to Galcanezumab in Patients With Episodic or Chronic Migraine: Results From the EVOLVE-1, EVOLVE-2, and REGAIN Randomized, Double-Blind, Placebo-Controlled Studies. Headache 2019; 59: 192-204.
}

Diese Post-hoc-Analyse zeigt, dass v.a. Patienten mit einem partiellen Ansprechen im ersten oder zweiten Monat eine hohe Wahrscheinlichkeit haben, im Verlauf doch noch zu Respondern zu werden.

\section{Hintergrund}

Mit den neuen CGRP-(Rezeptor)-Antikörper sind erstmals Prophylaktika mit einem migränespezifischen Ansatz verfügbar. Einer der Vorteile dieser Substanzen ist das schnelle Ansprechen. Eine dem Placebo überlegene Wirkung konnte in den Studien im Mittel schon nach 1-2 Wochen gezeigt werden. Allerdings sind das Mittelwerte über viele Patienten. Die Frage ob eine Behandlung über 1 Monat bereits ausreichen könnte, um Responder und Non-Responder voneinander zu unterscheiden, war bisher offen.

\section{Zusammenfassung}

Es handelt sich um eine Post-hoc-Analyse aus den großen Zulassungsstudien für den CGRP-Antikörper Galcanezumab. Es wurde geprüft, ob Patienten mit episodischer oder chronischer Migräne, die nach dem ersten oder den ersten 2 Monaten unter Galcanezumab definitionsgemäß noch keine Responder waren, im weiteren Verlauf eine Response zeigten. Wegen unterschiedlicher Studiendesigns und Subgruppenanalysen liest sich die Auswertung etwas kompliziert. Die Studien zur episodischen Migräne gingen über 6 Monate, und Responder waren definiert als Besserung der Migränetage um $\geq 50 \%$, dagegen lief die Studie zur chronischen Migräne nur über 3 Monate, und Responder waren definiert als Besserung um $\geq 30 \%$.
Von 878 Patienten mit episodischer Migräne zeigten 49 \% nach 1 Monat eine Response (Besserung der Migränetage um $\geq 50 \%$ ). Von den 450 Non-Respondern wurden 191 im Mittel der folgenden 5 Behandlungsmonate doch noch zu Respondern, das entspricht $42 \%$ der initialen Non-Responder. Bei den Non-Respondern, die nach dem ersten Monat ein Teilansprechen mit Besserung um 30-50\% gezeigt hatten, lag dieser Prozentsatz noch höher (62\%), aber auch bei denen mit einer initialen Besserung von $<10 \%$ wurden noch $26 \%$ zu Respondern. Von den Non-Respondern nach dem 2. Monat $(n=290)$ wurden immer noch $29 \%$ im Mittel der Monate 3-6 zu Respondern.

Von 555 Patienten mit chronischer Migräne zeigten $45 \%$ nach 1 Monat eine Response (für die chronische Migräne definiert als Besserung der Migränetage um $\geq 30 \%$ ). Von den 306 Non-Respondern wurden 74 im Mittel über die folgenden 2 Behandlungsmonate doch noch zu Respondern, das entspricht $24 \%$ der initialen Non-Responder. Wiederum war der Anteil bei denen mit partiellem Ansprechen (Besserung um 10-30\%) höher (bei 38\%) als bei denen mit Besserung < $10 \%$ (bei 15\%).

\section{Kommentar}

Diese Auswertung ist absolut zu begrüßen, da sie einem, jenseits der üblichen Analyse von Mittelwerten zu vordefinierten Zeitpunkten, Informationen über die Wahrscheinlichkeit des Ansprechens einzelner Patienten im Verlauf der Therapie gibt. Die Daten legen nahe, dass ein bedeutsamer Prozentsatz von Patienten mit Non-Response im 1. und/oder 2. Monat im Verlauf von 3 oder evtl. sogar 6 Monaten doch noch auf die Therapie mit Galcanezumab anspricht. Leider wurde die notwendige oder sinnvolle Dauer des Therapieversuchs nicht direkt analysiert. Erwartungsgemäß war bei Patienten mit einem teilweisen Ansprechen in Monat 1 die Chance auf ein volles Ansprechen im Laufe der weiteren Therapie höher als bei denen mit fehlendem initialen Ansprechen. Ein direkter Vergleich der Response im Verlauf zwischen episodischer und chronischer Migräne ist aus den vorliegenden Daten nicht möglich.

Einschränkend muss man sagen, dass die präsentierten Post-hoc-Analysen nicht im
Vergleich zu Placebo durchgeführt wurden. Dies ist aufgrund des Studiendesigns zugegebenermaßen schwierig (es gab ja keine Patienten, die im 1. Monat Verum und danach Placebo bekamen) aber lässt trotzdem offen inwieweit die im Verlauf noch zu Respondern gewordenen Patienten dies auch unter Placebo geworden wären.

Eine weitere interessante Frage, die vielleicht in folgenden Studien bearbeitet werden könnte ist, wie wahrscheinlich bei initialem Ansprechen ein im Verlauf anhaltendes Ansprechen ist.

Ruth Ruscheweyh, München

\section{Migräne und Kleinhirn}

\author{
**** Mehnert J, May A. Functional and structural \\ alterations in the migraine cerebellum. J Cereb \\ Blood Flow Metab 2017 doi: \\ $10.1177 / 0271678 \times 17722109$
}

Verschiedene Anteile des Kleinhirns sind bei Patienten mit einer Migräne vermehrt aktiv und auch die Verbindungen des Kleinhirns mit kortikalen Arealen sind unterschiedlich. Diese Befunde könnten auf eine Kontrollfunktion des Kleinhirns bei der Migräne hinweisen.

\section{Zusammenfassung}

Die Arbeitsgruppe um A. May, Hamburg, hat sich in den letzten Jahren vermehrt um die Charakterisierung der Aktivität von Hirnregionen im fMRI bei nozizeptiven Reizen bzw. bei Patienten mit Migräne gekümmert. Lange Jahre war wiederholt eine Aktivierung von Kleinhirnstrukturen bei Schmerzparadigmen im fMRI berichtet, aber nicht weiter diskutiert worden. In der hier vorgelegten Arbeit wurden insgesamt 54 Patienten mit einer Migräne zu verschiedenen Zeitpunkten des Migränezyklus (iktal, präiktal, interiktal) kernspintomografisch untersucht. Dabei wurde neben einer strukturellen Bildgebung (voxel-based morphometry, VBM) auch ein etabliertes funktionelles Paradigma untersucht: Applikation eines schmerzhaften chemischen Reizes im linken Nasenloch (Ammoniak) versus Rosenduft bzw. Luft. Die Ergebnisse der Patienten wurden dabei noch mit den Parametern Schmerzintensität, Alter, 
Häufigkeit der Attacken und Zeitpunkt innerhalb des Migränezyklus korreliert und mit den Ergebnissen von 54 altersgematchten Kontrollen verglichen.

Wesentliche Ergebnisse waren, dass sich die Befunde, die vorher schon bei Gesunden erhoben werden konnten, auch bei der Gruppe der Patienten mit Migräne bestätigen ließen. Darüber hinaus fanden sich bei den Patienten mit Migräne im Vergleich eine Abnahme der grauen Substanz in mehreren kortikalen Arealen Gyrus präcentralis, postcentralis, supramarginalis und links Gyrus angularis sowie Zunahme im Gyrus angularis rechts, im linken Pallidum und temporo-occipital. Dagegen fand sich im Hirnstamm und Kleinhirn nur eine Zunahme der grauen Substanz und zwar in den Kleinhirnarealen VI, VIIb, VIIla sowie Crus 1 und 2. Möglicherweise hat die Dauer der Erkrankung mit einer Abnahme in Areal V korreliert.

Bezüglich der funktionellen Daten zeigte sich bei trigeminaler Schmerzreizung wie erwartet eine Aktivierung im spinalen Trigeminuskern ipsilateral sowie im PAG und im ipsilateralen Crus I (Kleinhirn). Darüber hinaus war die funktionelle Verbindung des Kleinhirns (Crus) mit dem linken Thalamus, bilateral occipital und dem Gyrus fusiformis bei Patienten mit Migräne reduziert. Die verschiedenen Migränephasen zeigten einen Einfluss auf die Aktivität zerebellärer Strukturen wie z. B. verschiedene Abschnitte des Vermis.

Die Autoren diskutieren diese Ergebnisse als Hinweis darauf, dass bei der Migräne eine veränderte schmerzevozierte Aktivität, aber strukturelle Unterschiede im Kleinhirn verglichen mit Gesunden bestehen und dass möglicherweise die vermehr- te Aktivität als Kompensation einer verminderten Konnektivität des Kleinhirns zu kortikalen schmerzverarbeitenden Strukturen zu interpretieren ist. Dabei zeigt das Kleinhirn normalerweise einen inhibitorischen Einfluss auf die kortikalen Areale. Letztlich könnte diese verminderte kortikale Inhibition durch das Kleinhirn die Triggerung einer Migräneattacke durch eine kortikale Aktivitätsänderung erleichtern.

\section{Kommentar}

Es zeigt sich einmal mehr, dass das Kleinhirn vermutlich an vielen Prozessen beteiligt ist, welche bisher nicht dem Kleinhirn zugeordnet wurden. Letztlich hat sich das Kleinhirn in der Evolution parallel zu der Entwicklung der Großhirnhemisphären vergrößert und zeigt eine reziproke Verbindung mit vielen Großhirnarealen. Auch ist das Kleinhirn eine der Hirnstrukturen mit der größten Dichte von CGRP-Rezeptoren und im Kleinhirn wird auch der $\mathrm{Ca}^{2+-}$ Kanal CACNA1A, der bei der FHM Typ 1 mutiert ist, stark exprimiert. Der Ausgang des Kleinhirns, die Purkinjezellen, sind inhibitorisch, sodass von einer Hemmung nachgeschalteter Strukturen auszugehen ist. Die vorliegende Arbeit erweitert unser Wissen über die Beteiligung und die Anatomie des Kleinhirns bei der Migräne. Wir können aber zurzeit noch nicht sagen, welchen Prozess konkret das Kleinhirn im Rahmen eines Migränezyklus beeinflusst. Interessant wäre auch eine Untersuchung, ob Patienten mit einem Purkinjezellverlust z. B. durch Antikörper eine Veränderung vorbestehender Kopfschmerzen berichten. Letztlich sollte die Arbeit Anreiz sein, weitere Verbindungen des Kleinhirns mit Nozizeption zu untersuchen.

Andreas Straube, München

\section{INFORMATION}

***** Exzellente Arbeit, die bahnbrechende Neuerungen beinhaltet oder eine ausgezeichnete Übersicht bietet

**** Gute experimentelle oder klinische Studie

*** Gute Studie mit allerdings etwas geringerem Innovationscharakter

** Studie von geringerem klinischen oder experimentellen Interesse und leichteren methodischen Mängeln

* $\quad$ Studie oder Übersicht mit deutlichen methodischen oder inhaltlichen Mängeln

Die Kopfschmerz-News werden betreut von: Priv.-Doz. Dr. Ruth Ruscheweyh, Klinik und Poliklinik für Neurologie, Klinikum der Universität München, Marchioninistr. 15, 81377 München, Tel. 089/440073907, ruth. ruscheweyh@med.uni-muenchen.de

Die Besprechungen und Bewertungen der Artikel stellen die Einschätzung des jeweiligen Autors dar, nicht eine offizielle Bewertung durch die Deutsche Migräne- und Kopfschmerzgesellschaft. 